OPEN ACCESS

Edited by:

Vasyl Vasko,

Uniformed Services University of the

Health Sciences, United States

Reviewed by:

Melissa G. Lechner,

UCLA David Geffen School of

Medicine, United States

Ejigayehu Gigi Abate,

Mayo Clinic, United States

*Correspondence:

Renfei Wang

roslyn_en@163.com

${ }^{\dagger}$ These authors share first authorship

Specialty section:

This article was submitted to

Thyroid Endocrinology,

a section of the journal

Frontiers in Endocrinology

Received: 18 July 2021

Accepted: 18 October 2021

Published: 09 November 2021

Citation:

Yu F, Li X, Ji Y, Tan J, Zhang G, Wang $P$, He $Y$ and Wang $R$ (2021) Delayed Initial Radioiodine Adjuvant

Therapy Does Affect Biochemical Response in Intermediate- to HighRisk Differentiated Thyroid Cancer.

Front. Endocrinol. 12:743310.

doi: 10.3389/fendo.2021.743310

\section{Delayed Initial Radioiodine Adjuvant Therapy Does Affect Biochemical Response in Intermediate- to High- Risk Differentiated Thyroid Cancer}

\author{
Feng $\mathrm{Yu}^{1 \dagger}$, Xue $\mathrm{Li}^{1+}$, Yanhui $\mathrm{Ji}^{1}$, Jian Tan ${ }^{1}$, Guizhi Zhang ${ }^{1}$, Peng Wang ${ }^{1}$, \\ Yajing $\mathrm{He}^{1}$ and Renfei Wang ${ }^{1,2 *}$ \\ ${ }^{1}$ Department of Nuclear Medicine, Tianjin Medical University General Hospital, Tianjin, China, ${ }^{2}$ Department of Nuclear \\ Medicine, Shanghai Jiao Tong University Affiliated Sixth People's Hospital, Shanghai, China
}

Background: There are no definite recommendations on the optimal time of initiating radioactive iodine (RAl) therapy for differentiated thyroid cancer (DTC) patients in current relevant guidelines. This study aimed to investigate the relationship between the timing of initiating radioiodine adjuvant therapy (RAT) and the clinical outcomes based on dynamic follow-ups and assessments in intermediate- to high-risk DTC patients.

Methods: A total of 206 patients with intermediate- to high-risk DTC receiving RAT of 150 $\mathrm{mCi}$ were retrospectively reviewed. According to the time interval (TI: between thyroidectomy and initial RAT), the patients were divided into 2 groups: Group 1: $\mathrm{Tl}<3$ months ( $n=148)$, and Group 2: $\mathrm{TI} \geq 3$ months $(n=58)$. The RAT therapy response was evaluated as excellent response (ER), indeterminate response (IDR), biochemical incomplete response (BIR), structural incomplete response (SIR). The univariate and multivariate analyses were conducted to screen out factors associated with incomplete response $(I R=B I R+S I R)$. Finally, the prognostic nomogram was used to explain $I R$ rates as a valuable tool in clinical practice.

Results: Response to initial RAT was significantly different between 2 groups during dynamic follow-ups (all $\mathrm{P}<0.05$ ). Group 2 had significantly lower ER rates (37.9 vs 63.5, 52.0 vs 73.9, 64.4 vs 80.3, all $P<0.05$, respectively) and higher IR rates (39.7 vs 14.9, 36.0 vs 9.7, 12.2 vs 3.9 , all $P<0.05$, respectively) than group 1 during dynamic follow-ups. By univariate and multivariate analyses, prolonged TI (HR: 6.67, 95\% Cl: 2.241-19.857, $P=0.001$ ), soft tissue invasion (HR: $7.35,95 \% \mathrm{Cl}: 1.624-33.296, P=0.010)$, higher $\mathrm{sTg}$ (HR: $7.21,95 \% \mathrm{Cl}: 1.991-26.075, P=0.003$ ) were manifested to be independent risk factors for IR. The nomogram showed that soft tissue invasion, sTg, and TI were the top 3 contributors to the $\mathbb{I R}$.

Conclusions: Early RAT is associated with greater biochemical response but has no impact on SIR. Delayed initial RAT ( $\geq 3$ months after thyroidectomy) related to IR in intermediate- to high-risk DTC.

Keywords: Differentiated thyroid carcinoma (DTC), Radioiodine therapy, timing, therapy response, prognosis 


\section{INTRODUCTION}

The therapy methods of differentiated thyroid cancer (DTC) mainly include surgery, radioactive iodine (RAI) therapy, and thyrotropin (TSH) suppression therapy (1). Among them, postoperative selectivity RAI therapy is an essential supplement after thyroidectomy, playing a critical role in the purge of potential residual thyroid cancer and the therapy of distant metastases (2). According to the different purposes of RAI therapy, it mainly includes radioiodine remnant ablation (RRA), radioiodine adjuvant therapy (RAT) and radioiodine therapy of known disease (RTKD).

The timing of initiating the first post-thyroidectomy RAI therapy does matter for planning therapy when patients require this therapeutic modality (3-5). It varies around the world and may be affected by many factors, such as personal condition, social environment, and disease-related condition. To date, however, the optimal time of initiating RAI therapy remains not clear and there are no definite recommendations in current relevant guidelines $(1,6)$. Several studies have noted this tissue with different conclusions. Li et al (3) reported that delayed initial RRA ( $\geq 3$ months after thyroidectomy) related to incomplete response (IR) in low- to intermediate-risk DTC. On the other hand, Kim et al (7) showed that delaying the first RTKD until 6 months after total thyroidectomy had no impact on restaging, recurrence and mortality in intermediate-/high-risk PTC. However, previous studies usually enrolled patients with different disease status, and they received a variable dosage of RAI for a different purpose, consequently, the results may be incomparable between studies.

With the re-recognition of the clinical value of RAI therapy, the number of DTC patients with higher serum stimulated thyroglobulin (sTg) level or poor clinicopathologic features who received the first RAT gradually increases, while the number of DTC patients who received RRA gradually decreases. Moreover, most previous studies observed clinical outcomes based on disease recurrence and mortality, not the new therapy response system proposed by the American Thyroid Association (ATA) guideline (version 2015) that divided clinical outcomes into four scenarios, including excellent response (ER), indeterminate response (IDR), biochemical incomplete response (BIR), structural incomplete response (SIR) (8-11). In addition, we noticed that most previous studies drew their conclusions just based on final follow-up rather than dynamic follow-ups.

In this study, we tried to investigate the relationship between the timing of initiating RAT and the clinical outcomes based on dynamic follow-ups and assessments in intermediate- to highrisk DTC patients.

\section{MATERIALS AND METHODS}

The Ethics Committee approved the research of the Tianjin Medical University General Hospital. Written informed consent was given by all patients participating in the study. All clinical data used in this research were anonymized for analysis.

\section{Patients}

We retrospectively collected the clinical records of patients with DTC treated in our department (Department of Nuclear Medicine, Tianjin Medical University General Hospital, Tianjin, China) from October 2016 to March 2021. All these DTC patients underwent total thyroidectomy and received the first post-thyroidectomy RAT. Patients with intermediate- to high-risk and received RAT dose of $150 \mathrm{mCi}$ were included according to the ATA guidelines (version 2015), while individuals had poor clinicopathologic features that mainly contained anyone of the following characteristics: (i) with T4 status of tumor in situ, (ii) with soft tissue invasion, (iii) with cervical lymph node metastasis of $3 \mathrm{~cm}$ or more in diameter and (or) extranodal invasion, (iv) with serum $\mathrm{sTg}>10 \mathrm{ng} / \mathrm{mL}$. Patients were excluded with anyone of the followings: (i) with tumor of incomplete resection, (ii) with known distant metastases, (iii) post-operative serum Tg level highly suggestive of distant metastases, (iv) positive anti-Tg antibodies (TgAb) (>20 IU/mL, reference value: $0-40 \mathrm{IU} / \mathrm{mL}$ ), (v) missing critical data. Finally, 206 eligible DTC patients (139 women and 67 men, ratio 2.1:1) were enrolled in this study. The median age was 48 for women and 46 for wen. The flow chart was showed in Figure 1.

\section{Collection of Clinical Data}

The patients' clinical records including gender, age, date of surgery and initial RAT, TSH, sTg, TgAb before RAT and during the dynamic follow-ups, were thoroughly reviewed. The size and status of in-situ tumors and metastatic lymph nodes were obtained from operative recordings and postoperative pathological reports, and then, patients were staged according to the American Joint Cancer Committee (AJCC)/Union for International Cancer Control (UICC) TNM staging system (8th edition). The recurrence risk of individuals was classified based on the ATA guidelines (version 2015).

\section{Procedures of RAT and Follow-up Protocol}

Subsequent RAT was conducted after a low-iodine diet for at least 2 weeks and thyroid hormone withdrawal until the TSH exceeded $30 \mu \mathrm{IU} / \mathrm{mL}$. During hospitalization, cervical ultrasound and chest computed tomography (CT) would be examined routinely. Whole-body RAI scans (WBSs) were performed 3 days after ${ }^{131} \mathrm{I}$ administration. Afterwards, patients took levothyroxine for thyrotropin suppression routinely. RAI therapy was re-conducted 6 months after RAT in patients if ${ }^{131}$ I-avid persistent/recurrence/metastatic DTC lesions were identified by post WBS.

Patients who had no overt structural/functional disease were followed up for more than one year, meanwhile, they must take levothyroxine regularly for TSH suppression therapy. The follow-up visit and possible modulation of levothyroxine dosage were conducted at 1-, 3-, 6- and 12-months post RAT except as otherwise indicated, then the interval was extended to 6-12 months. Patients were regularly followed up by physical examination and measurements of thyroid hormones, TSH, $\mathrm{Tg}$ 


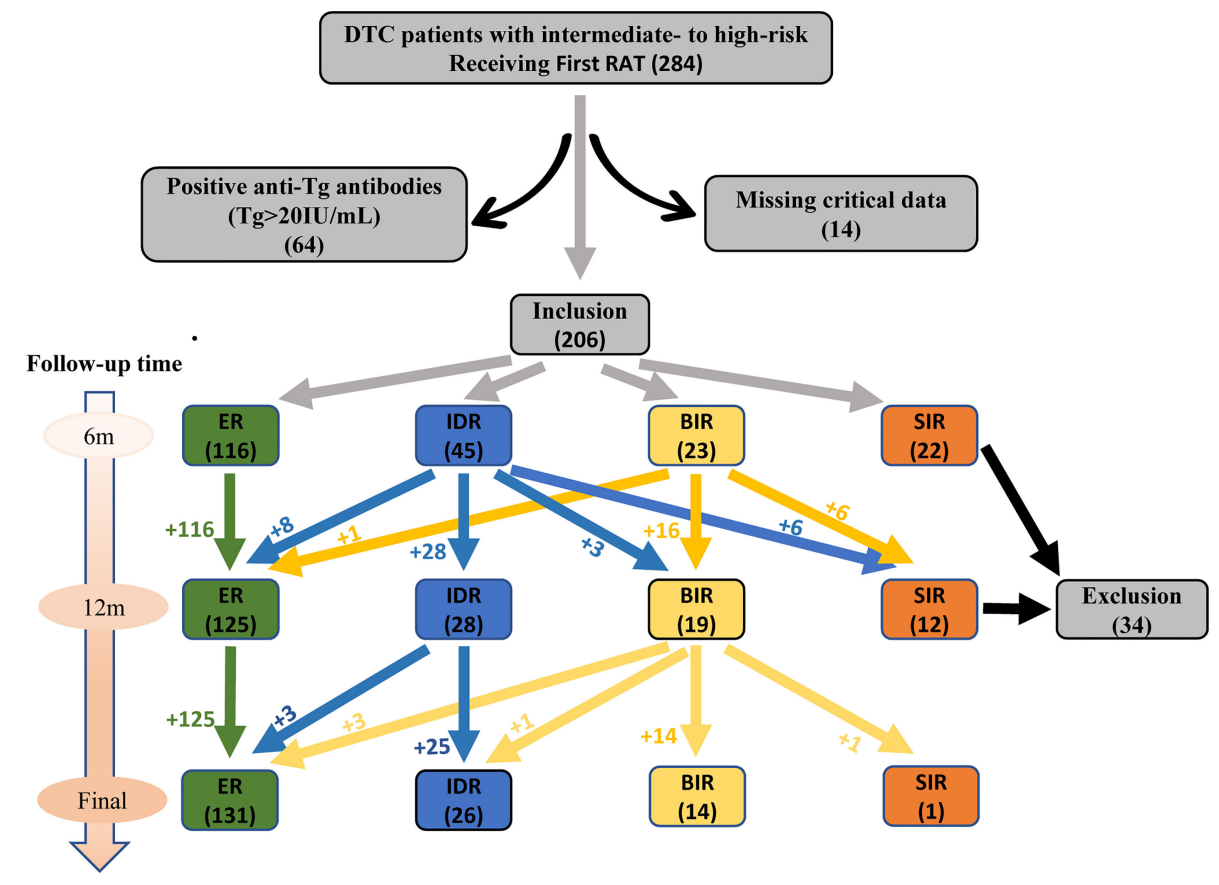

FIGURE 1 | Flow chart for selection process of eligible participants.

and TgAb. Neck ultrasound was also conducted during followup duration. Additional imaging studies (e.g., WBS, CT) were performed, as needed, whenever the clinical or laboratory findings raised the suspicion of persistent or recurrent disease.

\section{Study Design and Response Classifications}

All patients received RAT after total thyroidectomy. $6 \mathrm{~m}, 12 \mathrm{~m}$ after RAT and recently, we followed up these patients and evaluated the therapy response. According to the therapy stratification system, the therapy responses to RAT were assessed as either ER, IDR, BIR, or SIR at every follow-up. In addition, BIR and SIR were collectively referred to as incomplete response (IR).

Patients were categorized by their time interval (TI: between thyroidectomy and initial RAT) and divided into 2 groups: Group 1: TI $<3$ months ( $n=148)$, and Group 2: TI $\geq 3$ months $(n=58)$. Then, patients were further categorized by their clinicopathologic features and sTg level and divided into 3 sub-groups: poor clinicopathologic features (PCPF): DTC with poor clinicopathologic features and any sTg level, hyperthyroglobulinemia (hyper-Tg): DTC with sTg $>10$ $\mathrm{ng} / \mathrm{mL}$ and no poor clinicopathologic features, PCPF \& hyper-Tg: DTC with both poor clinicopathologic features and $\mathrm{sTg}>10 \mathrm{ng} / \mathrm{mL}$.

The therapy responses were evaluated between the two groups and the three sub-groups in dynamic follow-ups. Notably, the patients would be excluded from the later comparison of the responses who were evaluated as SIR, because these patients received consequent RAI therapy or other therapy. Then, the univariate and multivariate analyses were performed to explore the influence of some factors on patients' IR rate, such as age, gender, histological type, soft tissue invasion, $\mathrm{T}$ and $\mathrm{N}$ status, pre-therapy $\mathrm{TSH}, \mathrm{sTg}$, and recurrence risk. Finally, the nomogram was developed to explain IR rates for patients as a valuable tool in clinical practice.

\section{Statistical Analysis}

The Mann-Whitney $U$ test was used to compare differences in continuous variables, such as age, Pre-therapy TSH, sTg level, and follow-up duration. A chi-squared test was used to analysis differences in categorical variables, such as gender, histological type, T status, N status, AJCC stage, recurrence risk, soft tissue invasion, and structural or functional Disease existence (SoFD) in the first post-therapy of ${ }^{131} \mathrm{I}$ imaging. The univariate analysis was conducted by log-rank test. All $P$ values presented were 2tailed, and values $<0.05$ were considered to be statistically significant. Variables with statistically significant $P$ value in the univariate analysis were included in the Cox regression for independent risk analysis. The establishment of the nomogram was performed using $\mathrm{R}$ software (version 4.0.5; http://www.rproject.org/). All statistical analyses were performed using SPSS software version 25.0 (SPSS Inc., Chicago, IL, USA).

\section{RESULTS}

\section{Patients' Baseline Characteristics}

Table 1 showed the clinical and pathologic characteristics of group $1(n=148)$ and group $2(n=58)$. There were no significant differences in age, gender, histological type, $\mathrm{T}$ status, $\mathrm{N}$ status, AJCC stage, recurrence risk, soft tissue invasion, Pre-therapy 
TABLE 1 | Clinical and pathologic characteristics.

\begin{tabular}{|c|c|c|c|}
\hline Characteristics & $\begin{array}{c}\mathrm{TI}<3 \text { months } \\
(\mathrm{n}=148)\end{array}$ & $\begin{array}{c}\mathrm{TI} \geq 3 \text { months } \\
(\mathrm{n}=58)\end{array}$ & $\mathbf{P}$ \\
\hline Age at diagnosis & $46(33-55)$ & $46(35-55)$ & $0.825^{\mathrm{a}}$ \\
\hline \multicolumn{4}{|l|}{ Gender } \\
\hline Male & $44(29.7)$ & $23(39.7)$ & \multirow[t]{2}{*}{$0.171^{\mathrm{b}}$} \\
\hline Female & $104(70.3)$ & $35(60.3)$ & \\
\hline \multicolumn{4}{|l|}{ Histological type } \\
\hline Papillary & $145(98.0)$ & 57 (98.3) & \multirow[t]{2}{*}{$1.000^{\mathrm{C}}$} \\
\hline Follicular & $3(2.0)$ & $1(1.7)$ & \\
\hline \multicolumn{4}{|l|}{ T status } \\
\hline $\mathrm{T} 1$ & $51(34.5)$ & $15(25.9)$ & \multirow[t]{4}{*}{$0.494^{b}$} \\
\hline T2 & $11(7.4)$ & $5(8.6)$ & \\
\hline T3 & 35 (23.6) & $12(20.7)$ & \\
\hline T4 & 51 (34.5) & $26(44.8)$ & \\
\hline \multicolumn{4}{|l|}{$\mathrm{N}$ status } \\
\hline NO & $14(9.5)$ & $3(5.2)$ & \multirow[t]{3}{*}{$0.085^{b}$} \\
\hline $\mathrm{N} 1 \mathrm{a}$ & 77 (52.0) & $23(39.7)$ & \\
\hline $\mathrm{N} 1 \mathrm{~b}$ & $57(38.5)$ & $32(55.2)$ & \\
\hline \multicolumn{4}{|l|}{ AJCC stage } \\
\hline I & $110(74.3)$ & $45(77.6)$ & \multirow[t]{3}{*}{$0.696^{b}$} \\
\hline$\|$ & 19 (12.8) & $8(13.8)$ & \\
\hline III & 19 ( (12.8) & $5(8.6)$ & \\
\hline \multicolumn{4}{|l|}{ Recurrence Risk } \\
\hline Intermediate & $94(63.5)$ & $29(50.0)$ & \multirow[t]{2}{*}{$0.075^{\mathrm{b}}$} \\
\hline High & $54(36.5)$ & $29(50.0)$ & \\
\hline \multicolumn{4}{|l|}{ Soft tissue invasion } \\
\hline No & 77 (52.0) & $26(44.8)$ & \multirow[t]{2}{*}{$0.353^{b}$} \\
\hline Yes & $71(48.0)$ & $32(55.2)$ & \\
\hline Pre-therapy TSH ( $\mu \mathrm{lU} / \mathrm{mL})$ & $\begin{array}{c}68.89(50.52- \\
95.63)\end{array}$ & $69.40(50.62-96.74)$ & $0.994^{a}$ \\
\hline Stimulated Tg (ng/mL) & $\begin{array}{c}11.20(2.65- \\
20.70)\end{array}$ & $11.20(2.84-20.70)$ & $0.415^{a}$ \\
\hline \multicolumn{4}{|l|}{ SoFD } \\
\hline Yes & $18(12.2 \%)$ & $9(15.5 \%)$ & \multirow[t]{2}{*}{$0.830^{a}$} \\
\hline No & $130(87.8 \%)$ & 49 (84.5\%) & \\
\hline Follow-up duration (months) & $\begin{array}{l}22.1(10.23- \\
23.43)\end{array}$ & $22.8(17.23-28.53)$ & $0.400^{a}$ \\
\hline
\end{tabular}

Data are expressed as the median (percentiles 25-75) or frequencies.

SoFD means Structural or Functional Disease existence in the first post-therapy of ${ }^{131}$ I imaging.

a means Mann-Whitney U test.

${ }^{\boldsymbol{b}}$ means Pearson Chi-squared test.

${ }^{c}$ means Chi-squared test (continuity correction).
TSH, sTg level, SoFD, and follow-up duration between the two groups.

\section{Comparison of Responses to Initial RAT Between 2 Groups During Dynamic Follow-ups}

As showed in Table 2, the proportions of ER, IDR, BIR, SIR, Non-ER, IR, Non-IR were statistically different between the two groups (all $P<0.05$ ). With the extension of the follow-up period, the ER rates of both groups gradually increased, on the contrary, the IR rates gradually decreased. Besides, group 2 had significantly lower ER rates $(37.9$ vs $63.5,52.0$ vs 73.9, 64.4 vs 80.3, all $P<0.05$, respectively) and higher IR rates (39.7 vs 14.9, 36.0 vs $9.7,12.2$ vs 3.9 , all $P<0.05$, respectively) than group 1 during dynamic follow-ups. But apart from that, the Table 2 showed it had no significant difference in the proportions of SIR, Non-SIR between the two groups.

\section{Comparison of Responses to Initial RAT Between 2 Groups of Patients in Different Sub-Groups}

As showed in Table 3, all proportions of ER, IDR, BIR, SIR, NonER, IR, Non-IR in the 3 sub-groups respectively were statistically different between the two groups (all $P<0.05$ ). With the extension of the follow-up period, the ER rates of both groups in the 3 subgroups gradually increased, on the other hand, the IR rates gradually decreased. Besides, the sub-group PCPF showed that group 2 had significantly lower ER rates (36.2 vs 66.3, 51.2 vs $73.4,62.2$ vs 79.5 , all $P<0.05$, respectively) and higher IR rates (42.6 vs 12.9, 39.0 vs 10.6, 24.3 vs 4.5 , all $P<0.05$, respectively) than group 1 at the 3 different follow-up time points. Similarly in the left two sub-groups (with hyper-Tg or PCPF \& hyper-Tg), group 2 had significantly lower ER rates and higher IR rates during dynamic follow-ups (all $P<0.05$ ). Additionally, there were no significant difference in the proportions of SIR, Non-SIR between the two groups in the sub-group PCPF/hyper-Tg/PCPF \& hyper-Tg.

TABLE 2 | Comparison of response to initial RAT between 2 groups [n (\%)].

\begin{tabular}{|c|c|c|c|c|c|c|c|c|c|}
\hline \multirow[t]{2}{*}{ Response } & \multicolumn{2}{|c|}{$6 m$ follow-up } & \multirow[t]{2}{*}{$\mathbf{P}$} & \multicolumn{2}{|c|}{$12 m$ follow-up } & \multirow[t]{2}{*}{$\mathbf{P}$} & \multicolumn{2}{|c|}{ Final follow-up } & \multirow[t]{2}{*}{$\mathbf{P}$} \\
\hline & $\mathrm{TI}<3 \mathrm{~m}(\mathrm{n}=148)$ & $\mathrm{TI} \geq 3 \mathrm{~m}(\mathrm{n}=58)$ & & $\mathrm{TI}<3 \mathrm{~m}(\mathrm{n}=134)$ & $\mathrm{TI} \geq 3 \mathrm{~m}(\mathrm{n}=50)$ & & $\mathrm{TI}<3 \mathrm{~m}(\mathrm{n}=127)$ & $\mathrm{TI} \geq 3 \mathrm{~m}(\mathrm{n}=45)$ & \\
\hline ER & $94(63.5)$ & $22(37.9)$ & $0.001^{b}$ & 99 (73.9) & $26(52.0)$ & $0.000^{d}$ & $102(80.3)$ & $29(64.4)$ & NA \\
\hline IDR & 32 (21.6) & $13(22.4)$ & & $22(16.4)$ & $6(12.0)$ & & $20(15.7)$ & 6 (13.3) & \\
\hline $\mathrm{BIR}$ & $8(5.4)$ & 15 (25.9) & & $6(4.5)$ & $13(26.0)$ & & $4(3.1)$ & $10(22.2)$ & \\
\hline $\mathrm{SIR}$ & $14(9.5)$ & 8 (13.8) & & 7 (5.2) & 5 (10.0) & & $1(0.8)$ & $0(0)$ & \\
\hline ER & $94(63.5)$ & $22(37.9)$ & $0.001^{b}$ & 99 (73.9) & 26 (52.0) & $0.005^{b}$ & 102 (80.3) & $29(64.4)$ & $0.032^{b}$ \\
\hline Non-ER & $54(36.5)$ & $36(62.1)$ & & 35 (26.1) & 24 (48.0) & & 25 (19.7) & 16 (35.6) & \\
\hline $\mathbb{R}$ & $22(14.9)$ & $23(39.7)$ & $0.000^{b}$ & $13(9.7)$ & 18 (36.0) & $0.000^{b}$ & 5 (3.9) & $10(12.2)$ & $0.001^{c}$ \\
\hline Non-IR & $126(85.1)$ & 35 (60.3) & & $121(90.3)$ & $32(64.0)$ & & $122(96.1)$ & 35 (77.8) & \\
\hline SIR & $14(9.5)$ & 8 (13.8) & $0.452^{\mathbf{b}}$ & 7 (5.2) & 5 (10.0) & $0.406^{c}$ & $1(0.8)$ & $0(0)$ & NA \\
\hline Non-SIR & 134 (90.5) & $50(86.2)$ & & $127(94.8)$ & 45 (90.0) & & $126(99.2)$ & 45 (100.0) & \\
\hline
\end{tabular}

\footnotetext{
$\boldsymbol{b}_{\text {means Pearson Chi-squared test. }}$

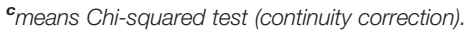

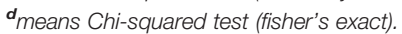

NA means not applicable.
} 
TABLE 3 | Comparison of responses to initial RAT between 2 groups in sub-groups [n (\%)].

\begin{tabular}{|c|c|c|c|c|c|c|c|c|c|}
\hline \multirow{2}{*}{$\begin{array}{l}\text { Response } \\
\text { PCPF }\end{array}$} & \multicolumn{2}{|c|}{$6 m$ follow-up } & \multirow[t]{2}{*}{$\mathbf{P}$} & \multicolumn{2}{|c|}{$12 m$ follow-up } & \multirow[t]{2}{*}{$\mathbf{P}$} & \multicolumn{2}{|c|}{ Final follow-up } & \multirow[t]{2}{*}{$\mathbf{P}$} \\
\hline & $\begin{array}{c}\mathrm{Tl}<3 \mathrm{~m} \\
\mathrm{n}=101\end{array}$ & $\begin{array}{l}\mathrm{Tl} \geq 3 \mathrm{~m} \\
\mathrm{n}=47\end{array}$ & & $\begin{array}{c}\mathrm{TI}<3 \mathrm{~m} \\
\mathrm{n}=94\end{array}$ & $\begin{array}{l}\mathrm{Tl} \geq 3 \mathrm{~m} \\
\mathrm{n}=41\end{array}$ & & $\begin{array}{c}\mathrm{TI}<3 \mathrm{~m} \\
\mathrm{n}=88\end{array}$ & $\begin{array}{l}\mathrm{Tl} \geq 3 \mathrm{~m} \\
\mathrm{n}=37\end{array}$ & \\
\hline ER & 67 (66.3) & $17(36.2)$ & $0.000^{c}$ & 69 (73.4) & $21(51.2)$ & $0.000^{d}$ & $70(79.5)$ & $23(62.2)$ & NA \\
\hline IDR & $21(20.8)$ & $10(21.3)$ & & 15 (16.0) & $4(9.8)$ & & 14 (15.9) & 5 (13.5) & \\
\hline $\mathrm{BIR}$ & $6(5.9)$ & $14(29.8)$ & & $4(4.3)$ & 12 (29.3) & & $3(3.4)$ & $9(24.3)$ & \\
\hline SIR & $7(6.9)$ & $6(12.8)$ & & $6(6.4)$ & $4(9.8)$ & & $1(1.1)$ & $0(0)$ & \\
\hline ER & 67 (66.3) & $17(36.2)$ & $0.001^{b}$ & 69 (73.4) & $21(51.2)$ & $0.012^{b}$ & 70 (79.5) & $23(62.2)$ & $0.042^{b}$ \\
\hline Non-ER & 34 (33.7) & 30 (63.8) & & 25 (26.6) & $20(48.8)$ & & $18(20.5)$ & $14(37.8)$ & \\
\hline $\mathrm{IR}$ & 13 (12.9) & 20 (42.6) & $0.000^{b}$ & 10 (10.6) & 16 (39.0) & $0.000^{b}$ & $4(4.5)$ & 9 (24.3) & $0.003^{c}$ \\
\hline Non-IR & $88(87.1)$ & $27(57.4)$ & & $84(89.4)$ & 25 (61.0) & & $84(95.5)$ & $28(75.7)$ & \\
\hline SIR & 7 (6.9) & $6(12.8)$ & $0.392^{b}$ & $6(6.4)$ & $4(9.8)$ & $0.741^{c}$ & $1(1.1)$ & $0(0)$ & NA \\
\hline Non-SIR & $94(93.1)$ & $41(87.2)$ & & 88 (93.6) & 37 (90.2) & & 87 (98.9) & 37 (100.0) & \\
\hline Hyper-Tg & $\mathrm{n}=80$ & $n=31$ & & $n=67$ & $n=24$ & & $n=63$ & $n=20$ & \\
\hline ER & $42(52.5)$ & $6(19.4)$ & $0.001^{b}$ & $46(68.7)$ & $7(29.2)$ & $0.000^{d}$ & $48(76.2)$ & $9(45.0)$ & NA \\
\hline IDR & $18(22.5)$ & 7 (22.6) & & 12 (17.9) & $4(16.7)$ & & $11(17.5)$ & $3(15.0)$ & \\
\hline $\mathrm{BIR}$ & $7(8.8)$ & $11(35.5)$ & & $5(7.5)$ & $9(37.5)$ & & $3(4.8)$ & $8(40.0)$ & \\
\hline SIR & 13 (16.3) & 7 (22.6) & & $4(6.0)$ & $4(16.7)$ & & $1(1.6)$ & $0(0.0)$ & \\
\hline ER & $42(52.5)$ & $6(19.4)$ & $0.002^{b}$ & $46(68.7)$ & $7(29.2)$ & $0.001^{b}$ & $48(76.2)$ & $9(45.0)$ & $0.009^{b}$ \\
\hline Non-ER & $38(47.5)$ & 25 (80.6) & & 21 (31.3) & $17(70.8)$ & & 15 (23.8) & $11(55.0)$ & \\
\hline $\mathrm{IR}$ & $20(25.0)$ & $18(58.1)$ & $0.001^{b}$ & $9(13.4)$ & $13(54.2)$ & $0.000^{c}$ & $4(6.3)$ & $8(40.0)$ & $0.001^{c}$ \\
\hline Non-IR & $60(75.0)$ & $13(41.9)$ & & 58 (86.6) & $11(45.8)$ & & $59(93.7)$ & $12(60.0)$ & \\
\hline SIR & 13 (16.3) & 7 (22.6) & $0.436^{b}$ & $4(6.0)$ & $4(16.7)$ & $0.243^{\mathrm{C}}$ & $1(1.6)$ & $0(0.0)$ & NA \\
\hline Non-SIR & 67 (83.8) & $24(77.4)$ & & 63 & 20 & & $62(98.4)$ & 20 (100.0) & \\
\hline PCPF\&Hyper-Tg & $n=33$ & $n=20$ & & $n=27$ & $n=15$ & & $n=24$ & $n=12$ & \\
\hline ER & 15 (45.5) & $1(5.0)$ & $0.004^{d}$ & 16 (59.3) & 2 (13.3) & $0.005^{d}$ & $16(66.7)$ & $3(25.0)$ & NA \\
\hline IDR & $7(21.2)$ & $4(20.0)$ & & $5(18.5)$ & $2(13.3)$ & & $5(20.8)$ & $2(16.7)$ & \\
\hline BIR & $5(15.2)$ & $10(50.0)$ & & $3(11.1)$ & 8 (53.3) & & $2(8.3)$ & 7 (58.3) & \\
\hline SIR & $6(18.2)$ & $5(25.0)$ & & $3(11.1)$ & $3(20.0)$ & & $1(4.2)$ & $0(0.0)$ & \\
\hline ER & $15(45.5)$ & $1(5.0)$ & $0.002^{b}$ & $16(59.3)$ & $2(13.3)$ & $0.004^{b}$ & $16(66.7)$ & $3(25.0))$ & $0.018^{b}$ \\
\hline Non-ER & $18(54.5)$ & 19 (95.0) & & $11(40.7)$ & $13(86.7)$ & & 8 (33.3) & $9(75.0)$ & \\
\hline IR & 11 (33.3) & $15(75.0)$ & $0.003^{b}$ & $6(22.2)$ & $11(73.3)$ & $0.001^{b}$ & $3(12.5)$ & 7 (58.3) & $0.012^{c}$ \\
\hline Non-IR & 22 (66.79) & $5(25.0)$ & & $21(77.8)$ & $4(26.7)$ & & $21(87.5)$ & $5(41.7)$ & \\
\hline SIR & $6(18.2)$ & $5(25.0)$ & $0.807^{c}$ & $3(11.1)$ & $3(20.0)$ & $0.649^{d}$ & $1(4.2)$ & $0(0.0)$ & NA \\
\hline Non-SIR & 27 (81.8) & $15(75.0)$ & & 24 & 12 & & $23(95.8)$ & $12(100.0)$ & \\
\hline
\end{tabular}

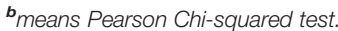

${ }^{c}$ means Chi-squared test (continuity correction).

PCPF means poor clinicopathologic features; Hyper-Tg means hyperthyroglobulinemia; PCPF\&Hyper-Tg means both PCPF and Hyper-Tg.

\section{Univariate and Multivariate Cox Analysis for Potential Risk Factors of IR to RAT}

Figure 2 and Table 4 showed the results of univariate Cox analysis about risk factors of IR to RAT. According to the univariate analysis, 6 significant variates including TI, T status, $\mathrm{N}$ status, soft tissue invasion, recurrence risk, and sTg level were risk factors for IR (all $P<0.05$ ), while there was no significant difference in other variables (all $P>0.05$ ). To further explore the predictive value, we entered these 6 risk factors into a multivariate Cox regression analysis model. The result showed that prolonged TI (HR: 6.67, 95\%CI: 2.241-19.857, $P=0.001$ ), soft tissue invasion (HR: 7.35, 95\%CI: 1.624-33.296, $P=0.010$ ), higher sTg (HR: 7.21, 95\%CI: 1.991-26.075, $P=0.003$ ) were manifested to be independent risk factors for IR.

\section{Prognostic Nomogram for IR Rates to RAT}

Based on the risk factors from univariate Cox analysis, we constructed a nomogram for predicting the IR rate. Each variable was assigned a score on a scale. By adding scores for each of the selected variables, a total score was obtained. Then a vertical line was dropped down from the total points row to estimate the risk of IR. The nomogram showed that soft tissue invasion, sTg, and TI were the top 3 contributors to the IR, followed by recurrence risk, $\mathrm{T}$ status, and N status (Figure 3).

\section{DISCUSSION}

RAI therapy for DTC has been used for a number of years, but the relationships between RAI initiating time and clinical outcomes are still not explicit. Recently, several studies have noted on this tissue with controversial conclusions. Scheffel et al. showed that the time ( $\geq 6$ months after thyroidectomy) between total thyroidectomy and RAI therapy does not interfere with the response to initial therapy, disease status at follow-up and recurrence rate in patients with DTC (8). Similarly, Kim et al (7) demonstrated that delaying the first RAI therapy until 6 months after total thyroidectomy had no impact on restaging, recurrence and mortality in intermediate- to high-risk DTC. However, Li et al (3) reported that delayed initial RRA $(\geq 3$ months after thyroidectomy) related to IR in low- to intermediate-risk DTC. However, the current DTC guidelines 

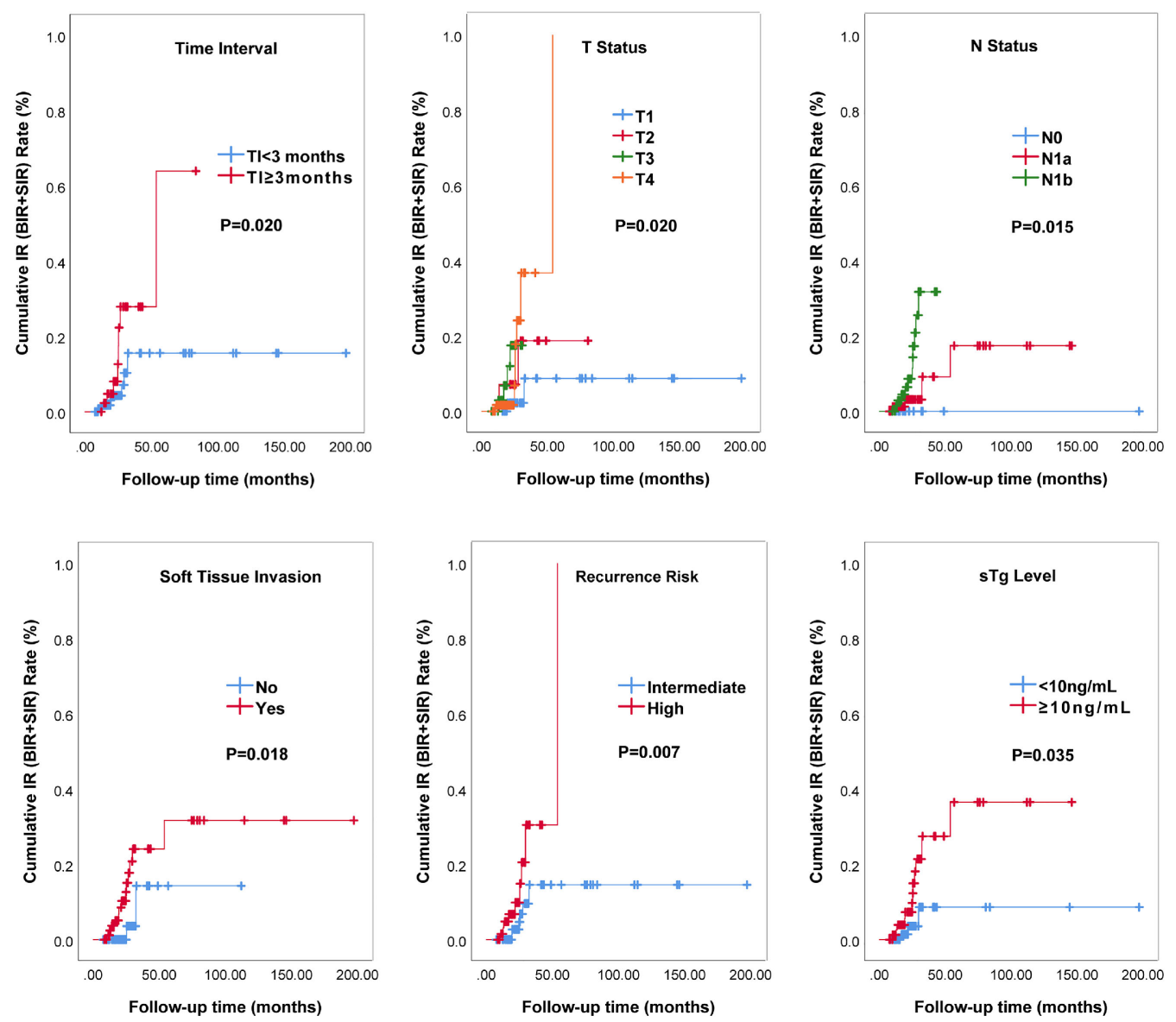

FIGURE 2 | IR curves of intermediate- to high-risk DTC patients receiving RAT analyzed by Kaplan-Meier test.

have no recommendations about the timing of RAI therapy (6, 12, 13). Accordingly, concerns about the best timing for RAI administration are shared among patients and physicians in clinical practice.

This study evaluated the impact of timing of the RAI therapy in intermediate- to high-risk patients with clinically characterized DTC. Notably, our study had something different from other studies. Firstly, the patients enrolled in our study all received first post-thyroidectomy RAT of potential residual tumor, neither RRA of remnant thyroid gland nor RTKD. Secondly, the therapy response was based on dynamic followups and assessments, not single checking-time points. Therefore, our findings provide evidence-based guidance on the appropriate timing of initial RAT administration in managing intermediateand high-risk patients with DTC.

We have evaluated the impact of the timing of RAT on intermediate- to high-risk DTC patients' outcomes followed by dynamic follow-ups at $6 \mathrm{~m}, 12 \mathrm{~m}$, and the last time after the first RAT. We showed that delayed initial RAT ( $\geq 3$ months after thyroidectomy) may cause higher IR rates in intermediate- to high-risk DTC patients during dynamic follow-ups. Our findings were contrast with those reported by Kim et al. and Scheffel et al, which significant methodological differences may partially explained. The therapy response evaluation methods used in these two studies mainly based on disease free survival (DFS) and overall survival (OS). However, OS is insufficient and inappropriate to assess the clinical outcomes of all DTC patients, especially for those with a wonderful 5-year OS of more than 95\% (14). Differently, we used the four-tiered therapy response evaluation system proposed by ATA guideline (version 2015), including ER, IDR, BIR and SIR. This new therapy response evaluation system confirmed that it could effectively predict the risk of recurrence and persistent disease, thereby providing dynamic risk estimates of responses to prior RAI therapy that can be used to tailor ongoing follow-up recommendations (15).

In our center, the DTC patients immediately took levothyroxine for thyrotropin suppression after thyroidectomy. One month after that, serum Tg, TgAb, thyroid hormones and neck ultrasound were measured and used to evaluate patients' status. Then, according to the patients' disease status and recurrence risk stratification, an individualized therapy strategy would be made for every patient. If necessary, subsequent RAT 
TABLE 4 | Univariate and multivariate Cox regression analysis based on all variables for IR.

Characteristics

Age at diagnosis
$\leq 45$
$>45$
Gender
Male
Female
Histological type
Papillary
Follicular
T status
T1
T2
T3
T4
N status
N0
N1a
N1b
AJCC stage
I
II
III
Recurrence Risk
Intermediate
High
Soft tissue invasion
No
Yes
Pre-therapy TSH ( $\mu l U / \mathrm{mL})$
$30-60$
$>60$
Stimulated Tg (ng/mL)
$<10$
$\geq 10$
SoFD
Yes
No
Time interval (month)
$<3$
$\geq 3$

Univariate analysis

HR (95\% Cl) P

$\mathbf{P}$

0.546

0.734

$1.197(0.424-3.377)$

Reference

21.187(0.000-10^7)

0.655

Reference

3.996 (0.551-28.981)

$11.237(1.810-69.755)$

$8.133(1.584-41.774)$

Reference

$1.436(0.763-27.562)$

2.436 (1.768-34.682)

Reference

$0.380(0.049-2.961)$

$1.813(0.394-8.334)$

Reference

3.918 (1.354-11.337)

Reference

5.080 (1.139-22.664)

1.184 (0.708-1.980)

Reference

3.587 (1.009-12.754)

Reference

1.562 (0.352-6.933)

Reference

5.623 (1.913-16.527)
Multivariate analysis

HR $(95 \% \mathrm{Cl})$
0.356

Reference

7.353 (1.624-33.296)

0.520

0.171

0.009

0.012

0.145

0.036

0.445

0.012

033

0.010

Reference

7.205 (1.991-26.057)

0.010

0.048

0.558

0.002
Reference $6.671(2.241-19.957)$

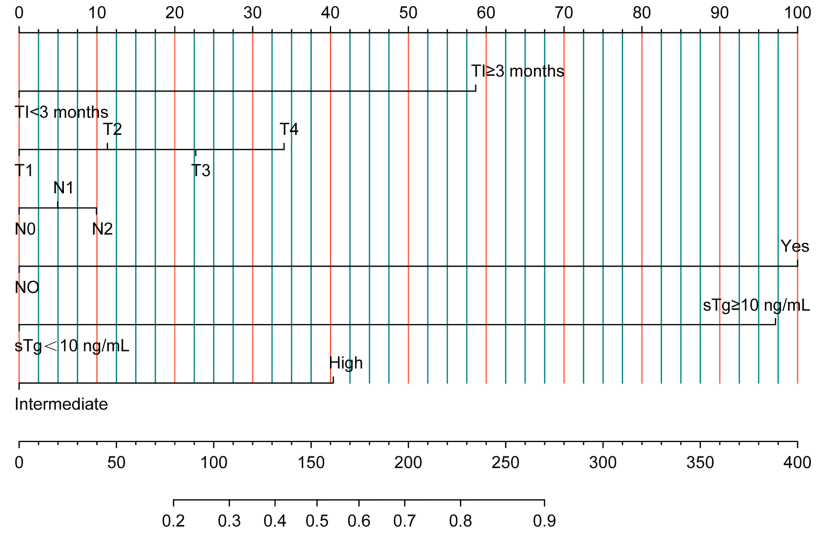

FIGURE 3 | Nomogram predicting IR rates for intermediate- to high-risk DTC patients receiving RAT. 
was conducted after a low-iodine diet for at least 2 weeks and thyroid hormone withdrawal until the TSH exceeded $30 \mu \mathrm{IU} /$ mL. Generally, most patients (accounts for about $71.8 \%$ of DTC patients during the study) completed their first RAT within three months after thyroidectomy, which may better reflect current practice patterns in the care of patients with DTC.

An earlier Meta-Analysis detailed that poor clinicopathologic features may relate to the occurrence of radioiodine refractory DTC (16). Tg reduction index and sTg level may be useful in predicting clinical outcomes and potential future disease recurrence in DTC (17-19). To further analyze the impact of poor clinicopathologic features and sTg level on response to initial RAT within two groups, we set three sup-groups based on clinicopathologic features and sTg level. The results showed that delayed initial RAT indicated higher IR rates during dynamic follow-ups, which was consistent with the general comparison of response to initial RAT between 2 groups.

Further univariate and multivariate analyses confirmed the TI ( $\geq 3$ months) to be an independent risk factor for IR. The IR rate of patients in the delayed group was 6.67 times higher than those who received RAT within three months. During the prolonged interval, the potential progression of microlesions may exist for a possible explanation of this observation, which was the possible mechanism in recurrence and distant metastatic DTC $(3,20)$. Furthermore, two other independent risk factors for IR, including soft tissue invasion and sTg level, were selected in multivariate Cox regression analysis. These findings were similar to Yang et al (21), who illustrated that extrathyroidal invasion was an important predictive factor $(\mathrm{P}=0.05)$ for $\mathrm{SIR}$, and higher preablative sTg and tumor size were independent predictive factors for $\operatorname{SIR}(\mathrm{P}<0.001$, $\mathrm{P}=0.002$, respectively). Additionally, different from Li et al (3), we demonstrated a significant relationship between soft tissue invasion/higher sTg level and IR. Different research populations might be responsible for these different results, as we only focused on intermediate- to high-risk DTC, while they only focused on low- to intermediate-risk DTC. Therefore, we suggested that prolonged TI, soft tissue invasion and higher sTg level might predict higher IR rates in patients with intermediate- to high-risk DTC receiving RAT. The patients with any of above three independent risk factors should need additional evaluations and possibly even complementary therapies.

In addition, to better determine the prognosis in intermediate- to high-risk DTC patients, we constructed a nomogram to predict IR rates based on 6 risk factors, including TI, T status, N status, soft tissue invasion, sTg level, and recurrence risk. From the nomogram, we could intuitively

\section{REFERENCES}

1. Chinese Society of Nuclear Medicine. Guidelines for Radioiodine Therapy of Differentiated Thyroid Cancer (2021 Edition). Chin J Nucl Med Mol Imaging (2021) 41(4):218-41. doi: 10.3760/cma.j.cn321828-20201113-00412

2. Cheng L, Sa R, Luo Q, Fu H, Jin Y, Tang L, et al. Unexplained Hyperthyroglobulinemia in Differentiated Thyroid Cancer Patients as an Indication for Radioiodine Adjuvant Therapy: A Prospective Multicenter Study. J Nucl Med (2021) 62(1):62-8. doi: 10.2967/jnumed.120.243642 see that soft tissue invasion, sTg level, and TI were the top 3 contributors to the IR, which were consistent with the results of multivariate Cox analysis.

Some limitations in our study must be considered. Firstly, the sample size was small. Secondly, the follow-up period was short and only RAI therapy responses were analyzed, but not DFS and recurrence rates. Thirdly, the exclusion of patients with SIR could cause data bias and the simple Chi-Square test may not be the most appropriate statistical approach when analyzing time dependent change. Finally, only a few patients (21/206) with TI more than 6 months were enrolled in our study, resulting in the impact of longer TI on IR cannot be assessed.

\section{CONCLUSIONS}

In conclusion, early RAT is associated with greater biochemical response but has no impact on SIR. Our study showed that prolonged TI ( $\geq 3$ months), Soft tissue invasion, and higher sTg level might predict higher IR rates in patients with intermediateto high-risk DTC receiving RAT. These patients may benefit from timely RAI therapy. The nomogram developed in this study may be a valuable tool when explaining IR rates to patients in clinical practice.

\section{DATA AVAILABILITY STATEMENT}

The raw data supporting the conclusions of this article will be made available by the authors, without undue reservation.

\section{ETHICS STATEMENT}

The studies involving human participants were reviewed and approved by The Ethical Committee of Tianjin Medical University General Hospital. The patients/participants provided their written informed consent to participate in this study.

\section{AUTHOR CONTRIBUTIONS}

FY and XL share first authorship. All authors contributed to the article and approved the submitted version. 
Patients With Intermediate-Risk Papillary Thyroid Cancer. J Clin Endocrinol Metab (2015) 100(4):1529-36. doi: 10.1210/jc.2014-4332

6. Haugen BR, Alexander EK, Bible KC, Doherty GM, Mandel SJ, Nikiforov YE, et al. 2015 American Thyroid Association Management Guidelines for Adult Patients With Thyroid Nodules and Differentiated Thyroid Cancer: The American Thyroid Association Guidelines Task Force on Thyroid Nodules and Differentiated Thyroid Cancer. Thyroid (2016) 26(1):1-133. doi: 10.1089/ thy.2015.0020

7. Kim M, Han M, Jeon MJ, Kim WG, Kim IJ, Ryu JS, et al. Impact of Delayed Radioiodine Therapy in Intermediate-/High-Risk Papillary Thyroid Carcinoma. Clin Endocrinol (Oxf) (2019) 91(3):449-55. doi: 10.1111/ cen. 14039

8. Scheffel RS, Zanella AB, Dora JM, Maia AL. Timing of Radioactive Iodine Administration Does Not Influence Outcomes in Patients With Differentiated Thyroid Carcinoma. Thyroid (2016) 26(11):1623-9. doi: 10.1089/ thy.2016.0038

9. Suman P, Wang CH, Moo-Young TA, Prinz RA, Winchester DJ. Timing of Adjuvant Radioactive Iodine Therapy Does Not Affect Overall Survival in Low- and Intermediate-Risk Papillary Thyroid Carcinoma. Am Surg (2016) 82(9):807-14.

10. Suman P, Wang CH, Abadin SS, Block R, Raghavan V, Moo-Young TA, et al. Timing of Radioactive Iodine Therapy Does Not Impact Overall Survival in High-Risk Papillary Thyroid Carcinoma. Endocr Pract (2016) 22(7):822-31. doi: 10.4158/ep151088.Or

11. Tsirona S, Vlassopoulou V, Tzanela M, Rondogianni P, Ioannidis G, Vassilopoulos C, et al. Impact of Early vs Late Postoperative Radioiodine Remnant Ablation on Final Outcome in Patients With Low-Risk WellDifferentiated Thyroid Cancer. Clin Endocrinol (Oxf) (2014) 80(3):459-63. doi: 10.1111/cen.12301

12. Rosário PW, Ward LS, Carvalho GA, Graf H, Maciel RM, Maciel LM, et al. Thyroid Nodules and Differentiated Thyroid Cancer: Update on the Brazilian Consensus. Arq Bras Endocrinol Metabol (2013) 57(4):240-64. doi: 10.1590/ s0004-27302013000400002

13. Cooper DS, Doherty GM, Haugen BR, Kloos RT, Lee SL, Mandel SJ, et al. Revised American Thyroid Association Management Guidelines for Patients With Thyroid Nodules and Differentiated Thyroid Cancer. Thyroid (2009) 19 (11):1167-214. doi: 10.1089/thy.2009.0110

14. Howlader NNA, Krapcho M. Seer Cancer Statistics Review, 1975-2013. Natl Cancer Institute (2016).

15. Tuttle RM, Tala H, Shah J, Leboeuf R, Ghossein R, Gonen M, et al. Estimating Risk of Recurrence in Differentiated Thyroid Cancer After Total Thyroidectomy and Radioactive Iodine Remnant Ablation: Using Response to Therapy Variables to Modify the Initial Risk Estimates Predicted by the
New American Thyroid Association Staging System. Thyroid (2010) 20 (12):1341-9. doi: 10.1089/thy.2010.0178

16. Luo Y, Jiang H, Xu W, Wang X, Ma B, Liao T, et al. Clinical, Pathological, and Molecular Characteristics Correlating to the Occurrence of Radioiodine Refractory Differentiated Thyroid Carcinoma: A Systematic Review and Meta-Analysis. Front Oncol (2020) 10:549882. doi: 10.3389/fonc.2020.549882

17. Barres B, Kelly A, Kwiatkowski F, Batisse-Lignier M, Fouilhoux G, Aubert B, et al. Stimulated Thyroglobulin and Thyroglobulin Reduction Index Predict Excellent Response in Differentiated Thyroid Cancers. J Clin Endocrinol Metab (2019) 104(8):3462-72. doi: 10.1210/jc.2018-02680

18. Prpić M, Franceschi M, Romić M, Jukić T, Kusić Z. Thyroglobulin as a Tumor Marker in Differentiated Thyroid Cancer - Clinical Considerations. Acta Clin Croat (2018) 57(3):518-27. doi: 10.20471/acc.2018.57.03.16

19. Yang X, Liang J, Li TJ, Yang K, Liang DQ, Yu Z, et al. Postoperative Stimulated Thyroglobulin Level and Recurrence Risk Stratification in Differentiated Thyroid Cancer. Chin Med J (Engl) (2015) 128(8):1058-64. doi: 10.4103/ 0366-6999.155086

20. Higashi T, Nishii R, Yamada S, Nakamoto Y, Ishizu K, Kawase S, et al. Delayed Initial Radioactive Iodine Therapy Resulted in Poor Survival in Patients With Metastatic Differentiated Thyroid Carcinoma: A Retrospective Statistical Analysis of 198 Cases. J Nucl Med (2011) 52 (5):683-9. doi: 10.2967/jnumed.110.081059

21. Yang X, Liang J, Li T, Zhao T, Lin Y. Preablative Stimulated Thyroglobulin Correlates to New Therapy Response System in Differentiated Thyroid Cancer. J Clin Endocrinol Metab (2016) 101(3):1307-13. doi: 10.1210/ jc.2015-4016

Conflict of Interest: The authors declare that the research was conducted in the absence of any commercial or financial relationships that could be construed as a potential conflict of interest.

Publisher's Note: All claims expressed in this article are solely those of the authors and do not necessarily represent those of their affiliated organizations, or those of the publisher, the editors and the reviewers. Any product that may be evaluated in this article, or claim that may be made by its manufacturer, is not guaranteed or endorsed by the publisher.

Copyright $\odot 2021 \mathrm{Yu}, \mathrm{Li}$, Ji, Tan, Zhang, Wang, He and Wang. This is an open-access article distributed under the terms of the Creative Commons Attribution License (CC BY). The use, distribution or reproduction in other forums is permitted, provided the original author(s) and the copyright owner(s) are credited and that the original publication in this journal is cited, in accordance with accepted academic practice. No use, distribution or reproduction is permitted which does not comply with these terms. 